\title{
Ethmoid Fungal Ball Causing Diplopia
}

\author{
Jong Yuap Seong, Tae Gu Kang, Dong Hoon Lee, and Sang Chul Lim* \\ Department of Otolaryngology-Head and Neck Surgery, Chonnam National University Hwasun Hospital, Chonnam National University \\ Medical School, Hwasun, Korea
}

An 81-year-old female visited our hospital with diplopia and proptosis via the Emergency room. She complained of diplopia a day prior when she looked to her right and her left eyeball couldn't move to the right. The light reflex was intact and proptosis was not prominent. An ophthalmologist checked her eyeballs and It seemed that she had a limitation of medial rectus movement of her left eyeball based on the Lancaster and diplopia chart. She had hypertension and diabetes mellitus without reaching an immunocompromised status. A physical examination of the nasal cavity, head, and neck was unremarkable. The patient did not have any nasal symptoms, such as nasal obstruction, rhinorrhea, nasal bleeding, or nasal swelling. Her chest x-ray was normal. To exclude a central nervous system problem, a brain magnetic resonance imaging (MRI) scan was conducted and no specific pathological findings were found except a low attenuated lesion in the left posterior ethmoid region using $\mathrm{T} 1$ imaging (Fig. 1). The computed tomography $(\mathrm{CT})$ scan showed a hazy lesion, approximately 1.0 $\mathrm{cm}$ in size, in the left posterior ethmoid. The findings also showed there were no erosive bony lesions and the lamina papyracea was intact (Fig. 1).

Various departments of our hospital, such as doctors of neurology and ophthalmology, collaborated to resolve the problem, but were unable to determine the correct final diagnosis. We regarded the diplopia as a rhinologic prob- lem and performed a functional endoscopic sinus surgery. After we reached the left posterior ethmoid, a fungal ball was found. The mucosa was relatively healthy (Fig. 2). The postoperative course was uneventful. Our patient's ophthalmic problem dramatically improved after the surgery. The histopathological analysis revealed a fungal ball of Aspergillosis, without mucosal invasion. The patient has not reported experiencing diplopia since the removal of the fungal ball from the ethmoid region. No evidence of recurrence was present during the last follow-up.

Usually, diseases in the sphenoid sinus cause ophthalmologic problems. ${ }^{1,2}$ This is due to the presence of the optic nerve, the oculomotor nerve, the trochlear nerve, the ophthalmic nerve, the trigeminal nerve, and the abducens nerve in this region. Consequently, there are few published reports about diplopia and related to sphenoid sinus diseases. $^{1,2}$

One case study claimed ethmoid disease had caused medial rectus muscle palsy. ${ }^{3}$ However, in that case, the patient had a history of trauma and the lamina papyracea was destroyed, whereas our patient's bone structure was intact. Therefore, we consider the cause of our patient's symptoms to be sinus pressure on the ethmoid bone. After removing the fungal material, the patient's ophthalmic problem was dramatically resolved.

In conclusion, to the best of our knowledge, there have
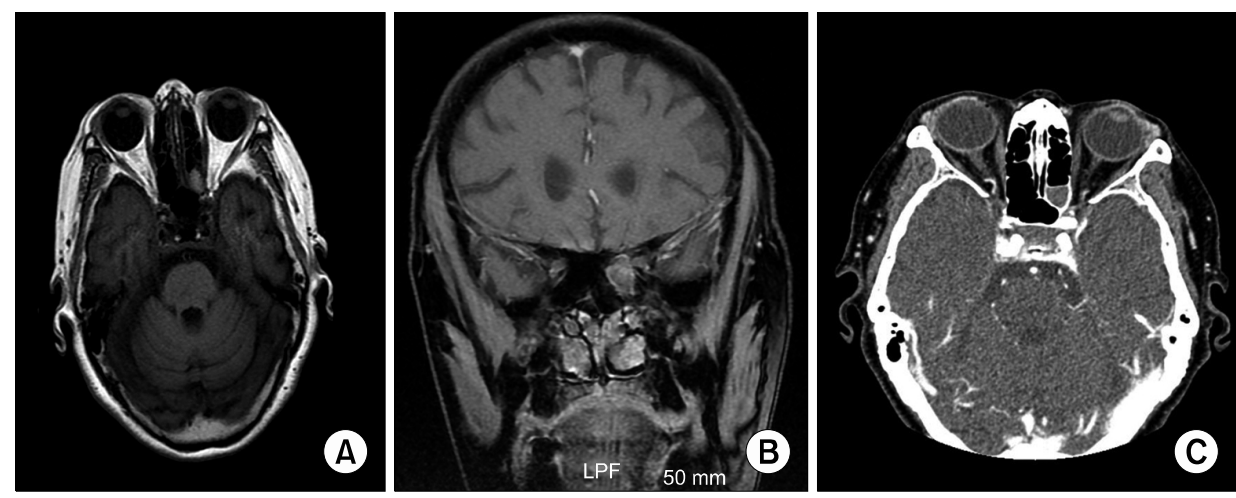

FiG. 1. Brain MRI T1 axial (A) and coronal (B) scan shows a low-attenuated lesion of approximately $1.2 \times 0.7 \mathrm{~cm}$ in the left posterior ethmoid sinus. (C) PNS CT enhanced axial scan shows a hazy lesion of approximately $1.0 \times 0.9 \mathrm{~cm}$ with a thickened bony structure. No erosive bone lesion was found.

\section{Corresponding Author: \\ Sang Chul Lim \\ Department of Otolaryngology-Head and Neck Surgery, Chonnam National University Hwasun Hospital, Chonnam National University Medical School, 322 Seoyang-ro, Hwasun-eup, Hwasun 58128, Korea \\ Tel: +82-61-379-8190, Fax: +82-61-379-8199, E-mail: limsc@chonnam.ac.kr}

Article History:

Received April 5, 2019

Revised April 18, 2019

Accepted May 3, 2019 

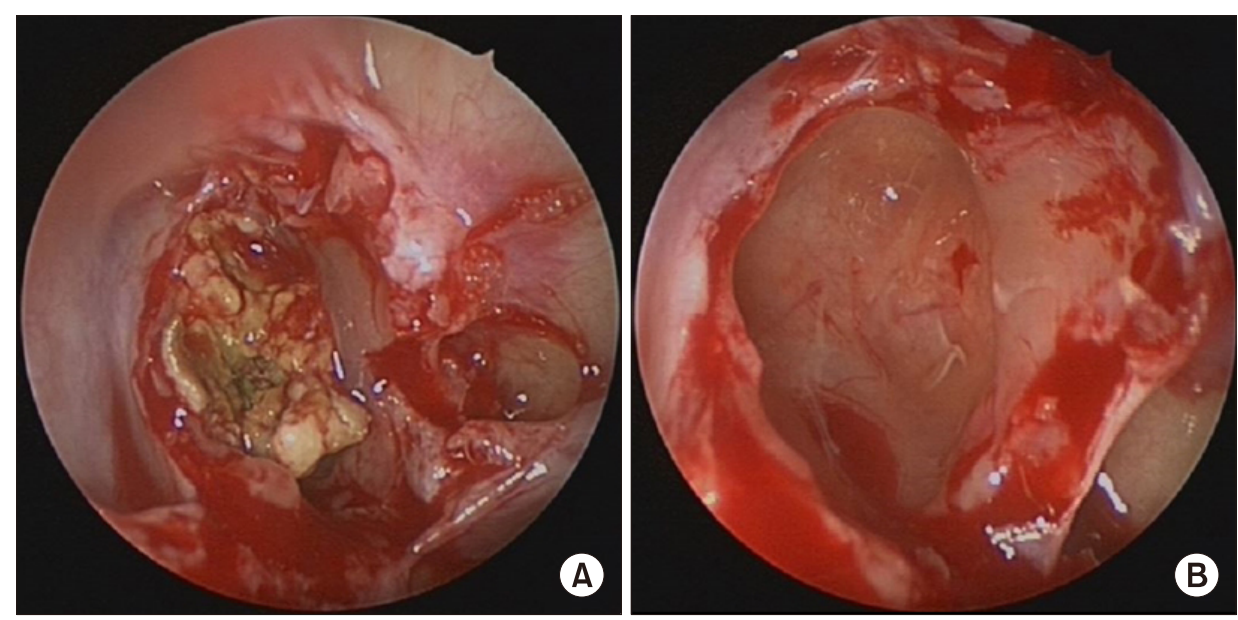

Fig. 2. (A) The presence of yellowish and thick fungal material in the left posterior ethmoid sinus. (B) After removing the fungal material, the mucosa was swollen, but relatively healthy.

not been any published studies regarding ethmoid fungal balls causing diplopia. We have presented an unusual case of a patient with an ethmoid fungal ball who suffered from diplopia. Functional endoscopic sinus surgery was used to successfully resolve the problem. ${ }^{4,5}$

\section{CONFLICT OF INTEREST STATEMENT}

None declared.

\section{REFERENCES}

1. Muneer A, Jones NS, Bradley PJ, Downes R. ENT pathology and diplopia. Eye (Lond) 1998;12:672-8.

2. Kim HJ, Shin SY, Kim SW, Cho JS. A case of fungal infection in the onodi cell with diplopia. Korean J Otorhinolaryngol-Head Neck Surg 2014;57:792-4.

3. Lee JH, Kang JS, Park KH, Park SI. Interesting cases of the ethmoid disease causing medial rectus muscle palsy. Korean J Otorhinolaryngol-Head Neck Surg 1988;31:852-6.

4. Yeo CK, Ahn BH, Kim JS, Kim YD, Shin SH, Ye MK. Fungal ball in sinus: multi-center study in Daegu. J Rhinol 2005;12:105-7.

5. Shim BS, Song YJ, Han KY, Kim JH, Ha MS, Kim JY. Clinical features of bilateral paranasal sinus fungus ball. J Rhinol 2010;17: 33-6. 